\title{
Lidil
}

Revue de linguistique et de didactique des langues

\section{Figures de l'auteur et hiérarchisation énonciative}

\section{Alain Rabatel et Francis Grossmann}

\section{OpenEdition}

Journals

Édition électronique

URL : https://journals.openedition.org/lidil/2153

DOI : 10.4000/lidil.2153

ISSN : 1960-6052

\section{Éditeur}

UGA Éditions/Université Grenoble Alpes

\section{Édition imprimée}

Date de publication : 1 juin 2007

Pagination : $9-23$

ISBN : 978-2-84310-100-7

ISSN : $1146-6480$

Référence électronique

Alain Rabatel et Francis Grossmann, « Figures de l'auteur et hiérarchisation énonciative », Lidil [En ligne], 35 | 2007, mis en ligne le 25 mars 2009, consulté le 12 mai 2021. URL : http:// journals.openedition.org/lidil/2153; DOI : https://doi.org/10.4000/lidil.2153

Ce document a été généré automatiquement le 12 mai 2021.

(C) Lidil 


\title{
Figures de l'auteur et hiérarchisation énonciative
}

\author{
Alain Rabatel et Francis Grossmann
}

Un bon lecteur est celui qui pratique des modes de lecture diversifiés, notamment à travers une grande mobilité énonciative, en gérant la diversité des origines énonciatives, en hiérarchisant les contenus propositionnels en fonction du degré de prise en charge ou de distanciation qu'il manifeste à leur égard, ainsi qu'à travers son aptitude à reformuler les points de vue en objets de savoir. Cette compétence est mise à l'épreuve à travers un type de lecture singulier: celui dont la finalité est l'appropriation de savoirs, et qui s'effectue à partir de supports dont le prototype reste, à l'école, le manuel. Une des questions cruciales qui se pose pour le jeune lecteur est sans doute d'être en mesure de (re)construire la cohérence du discours qu'on lui tient, que ce discours vise à décrire un objet de connaissance spécifique, ou qu'il vise, au-delà de cet objet, à développer un point de vue sur le monde. L'idée même de «lecture critique ", si souvent invoquée, n'a de sens que si la capacité de recul qu'on attend du lecteur se fonde d'abord sur la possibilité de faire sens, à partir d'un «centre interprétatif », permettant d'assigner un principe d'unité à l'hétérogène.

\section{Les évolutions récentes de la figure de l'auteur dans les manuels scolaire}

2 Or, à l'heure actuelle, et depuis deux petites décennies, on peut penser qu'un faisceau de causes converge pour réduire voire effacer le discours primaire enchâssant du savoir (comme celui qu'exprimait, pour l'histoire, le « Mallet et Isaac », prototype du manuel « républicain ») au profit de documents de toute nature (témoignages, analyses, reproduction d'œuvres d'art, schémas, graphiques, etc.). D'où la floraison de manuels (école primaire, collège, voire lycée) qui juxtaposent des documents provenant de sources énonciatives diverses, sans hiérarchisation, ou sans qu'une hiérarchisation soit clairement présentée au lecteur. Les traces d'un point de vue d'auteur peuvent cependant apparaître, par exemple dans le résumé qui termine la leçon. Il reste donc à 
vérifier, à travers des études de cas précises et documentées, si à cet éclatement de la surface textuelle et à l'hétérogénéité des documents, correspond aussi un effacement $\mathrm{du}$ discours primaire intégrateur. On peut au moins constater que cette nouvelle forme de «mélanges » textuels pose des problèmes nouveaux, tant du point de vue de la conception sémiographique des manuels, que du point de vue des contenus à transmettre.

3 Les manuels, influencés par les hyperstructures de la presse et par les réseaux hypertextuels de l'Internet, développent un mode de présentation qui fonctionne sur le mode de la double page, ou sur des modules de doubles-pages successives, selon la complexité de la leçon ou le niveau. Le recours à ce type de mise en page/mise en scène énonciative parait particulièrement tentant lorsqu'il s'agit d'exposer des évènements sur lesquels il y a conflit (colonisation, guerre d'indépendance, origine de l'homme, théories économiques), ou dont on postule qu'il peuvent susciter des points de vue antagonistes chez des groupes d'origines culturelles et ethniques différentes plus particulièrement sensibles à certaines problématiques, qui peuvent être présentées en histoire ou en littérature, telles le conflit israélo/palestinien, la pensée des Lumières du $\mathrm{XVIII}$ siècle et ses positions sur la religion, la tolérance, la place des femmes, etc. Ce problème n'est pas limité aux manuels ou aux livres du professeur, il concerne l'ensemble de la vulgarisation scientifique, et intègre des situations didactiques variées, cours magistraux, interactions autour de l'exploitation oralo-graphique des manuels dans la classe, etc. Cette évolution, si elle se confirme, pose donc la question de ce que l'on peut nommer «la figure de l'auteur » dans le champ didactique. Cette expression veut d'abord caractériser la manière dont l'instance qui, dans le texte, se présente comme responsable principal de l'énonciation, fournit au lecteur les éléments qui lui permettent de construire l'interprétation, à travers la gestion de la polyphonie et la hiérarchisation des points de vue ${ }^{1}$. Mais elle prend surtout en compte la manière dont il assume ce rôle et s'incarne dans le déroulement même du texte : l'auteur peut ainsi se montrer très présent ou plus effacé, se poser comme prudent, modeste ou péremptoire, polémique ou consensuel, être plus ou moins attentif à anticiper les questions que pourrait se poser le lecteur («xreader friendly » comme disent les Anglo-saxons) ou à l'inverse obscur et peu coopératif. Dans le cas qui nous occupe, c'est la multiplicité même des points de vue liés à la segmentation de l'espace textuel et à la profusion des documents qui viendrait en quelque sorte mettre en échec l'émergence d'un point de vue d'auteur ou, pour revenir à la métaphore, venir en brouiller la figure.

4 Certes, l'auteur en didactique, avec sa prétention à tenir un discours univoque et englobant, a pu susciter les critiques, comme en témoigne la remise en cause du «Lagarde et Michard», dans les années soixante-dix. Les évolutions, voire les débats scientifiques ont fait exploser toute prétention à rendre compte du complexe par un discours unilatéral. La didactique n'a pu que se saisir de la diversité des sources et des approches pour rendre compte du complexe. Le rejet d'un modèle didacticopédagogique descendant, transmissif et, concomitamment, la volonté pédagogique de mettre l'élève au travail en le faisant analyser des documents (socio-constructivisme, théories du conflit cognitif), l'influence des mises en pages dans les médias et dans les nouvelles technologies (hypertextes), la montée en puissance du relativisme, bref, tout un ensemble de phénomènes disparates participent à un titre ou à un autre à la disparition tendancielle du discours du maitre au profit de documents ou d'exercices supports d'activités au terme desquelles le savoir est à construire et doit faire l'objet 
d'une trace écrite parfois réduite à peu de choses, tant à l'école primaire qu'au collège. La tendance semble avoir été amplifiée par la massification du système scolaire, par l'intégration d'un nombre important d'enfants provenant d'autres cultures, dans tous les cas, par l'augmentation du nombre des enfants dont les liens avec la culture (scolaire) légitime étaient distendus et rendaient nécessaire le recours à une pédagogie plus active que le modèle transmissif (et donc aussi le recours à des manuels différents de ceux sur lesquels s'appuyait le modèle transmissif). On ne reviendra pas ici sur les débats qui, comme souvent en France, ont eu tendance à privilégier les oppositions frontales abstraites par rapport aux propositions concrètes : il nous semble souhaitable d'adapter le modèle transmissif classique et de l'ouvrir à la pluralité des références culturelles, sans pour autant renoncer à la cohérence des savoirs transmis, et sans participer à l'illusion pédagogiste d'un savoir que l'élève devrait, à lui seul, (re)construire ou (re)découvrir.

5 Ainsi, si de fortes évolutions sociales et culturelles ont heureusement convergé pour modifier les discours didactiques et les attitudes pédagogiques traditionnels, elles s'accompagnent aussi de retombées négatives. Ces pratiques peuvent parfois conduire à un relativisme qui nie toute possibilité de savoir et, sur des notions complexes ou dissensuelles, renvoie les thèses en présence aux opinions de groupes humains, en une sorte de démission devant l'ambition rationnelle de construire des savoirs étayés et argumentés, qui puissent faire l'objet d'appropriation commune indépendamment des origines ou des opinions: ainsi en est-il du bilan du stalinisme, de la politique impérialiste des Etats-Unis, de la (dé)colonisation ${ }^{2}$, pour prendre des exemples historiques. D'autres facteurs, apparemment plus circonstanciels et plus "locaux» (puisqu'ils concernent plus spécifiquement la tradition française) ont également joué un rôle: c'est le cas par exemple de l'affaissement du modèle de l'enseignement grammatical qui après avoir un temps été renouvelé par l'approche linguistique, semble aujourd'hui relégué à des fonctions purement instrumentales, "l'observation réfléchie de la langue » se voyant assigner une tâche à la fois réduite (puisqu'elle n'est plus qu'ancillaire) et démesurée (puisqu'on lui demande de guider la lecture et l'écriture des différentes formes de discours).

\section{Ce que deviennent les manuels scolaires dans l'interaction maitre-élève}

6 Même si notre propos concerne de manière centrale ce que l'on pourrait appeler l'auctorialité didactique, qu'elle concerne les manuels classiques ou les supports multimédia, cette question ne peut être traitée sans qu'on envisage aussi la chaîne de réception. Les usages que font les enseignants des manuels peuvent se conformer ou non au «mode d'emploi » préparé par les auteurs, mode d'emploi qui peut lui-même prévoir des marges de manœuvres plus ou moins importantes. Il y a un rapport dialectique entre la figure du lecteur et celle de l'auteur : cette dernière se dessine en effet également à travers les places assignées aux usagers (maitres et élèves). Inversement, les lecteurs du manuel peuvent, par ces chemins de traverse qu'évoque Michel de Certeau (1990), user plus ou moins librement du produit textuel qu'on leur destine. La question de la lisibilité des savoirs se joue aussi à travers cette évolution de la répartition des rôles, qui semble aboutir, tendanciellement, à renforcer le pôle du médiateur enseignant (sorte de superlecteur du manuel) par rapport à l'auteur, 
désormais davantage conçu comme un prestataire de services, offrant ici de la documentation, là une précision ou une définition.

7 Il nous semble donc qu'il y a un réel enjeu à proposer, aujourd'hui, une réflexion sur la manière dont sont donnés à lire les savoirs scolaires, confrontés à cette crise de lisibilité. Revenir sur les mises en scènes énonciatives à l'œuvre dans les manuels c'est, pour nous, tenter de comprendre comment la voix de l'auteur ou de l'enseignant qui se fait son porte- parole émerge de la diversité des sources énonciatives et opère la synthèse de l'hétérogène sans laquelle il n'est pas de compréhension effective.

8 On part donc de l'hypothèse que la figure de l'auteur est à même de favoriser la compréhension des lecteurs par la construction intratextuelle d'un locuteur/ énonciateur intratextuel qui serve de centre interprétatif (Maingueneau, 1990, Philippe, 2000) auquel les apprenants puissent s'identifier, et l'on cherchera à se demander comment les élèves, les parents et les enseignants hiérarchisent les informations, selon quels principes, et quelle image du sujet connaissant. Telle est la question à laquelle ce numéro de Lidil entend répondre. Il nous faut donc à présent préciser le sens qui sera donné ici aux notions d'« auteur » et de « figure de l'auteur ».

\section{Figures de l'auteur et remise en cause de la fonction auteur-créateur-fondateur}

9 La notion d'auteur, loin de correspondre à un donné naturel, est une construction opaque, tributaire de multiples médiations socio-culturelles, ainsi que Barthes et Foucault, notamment, l'ont montré.

10 Le premier rappelle que cette notion d'auteur est très récente dans l'histoire, en lien avec d'une part l'invention de l'imprimerie, et d'autre part un certain nombre d'évolutions politiques, sociales et culturelles qui ont favorisé son émergence aux alentours de la Renaissance. En effet, jusqu'au Moyen Âge, dans les communautés d'érudits rassemblés autour des textes recopiés par les copistes, la notion même d'auteur n'a guère de sens, et son statut, s'il existe, se rapproche de celui en vigueur dans les sociétés orales : on distingue le scriptor du commentator du compilator, mais pas d'auctor au sens moderne du mot. Ces différents rôles renvoient à un intellectuel collectif qui se fonde sur une pensée, elle aussi collective, se transmettant collectivement. C'est plus tard qu'émergent complémentairement les notions d'individu et d'auteur (Barthes 1970 : 184-185).

11 Le second a fortement mis en relief les modifications de la figure de l'auteur en lien avec les bouleversements dans les sciences humaines ou exactes : la figure de l'auteur, comme la fonction-auteur, change d'objectif, par rapport à l'approche traditionnelle de l'auteur-démiurge, au point que l'épistémologue ne peut plus appréhender la fonction auteur à partir de l'image d'un sujet originaire unique, tellement le caractère prégnant de l'interdiscours ainsi que la dimension interactionnelle des échanges remettent en cause le mythe de l'auteur-fondateur (Foucault 2001a : 838-839; cf. également Foucault 2001a : 543, 726 et 727 et Foucault 2001b : 28-40).

12 Toutes ces données sont significatives si l'on pense aux figures de l'auteur en contexte didactique. À l'écrit, l'auteur didacticien est le plus souvent un compilateur qui présente les savoirs sous une forme cumulative; il gomme ou estompe les conflits provenant des différents cadres historiques ou paradigmes scientifiques, se faisant le 
porte-voix d'une vision patrimoniale de savoirs naturalisés. À l'oral, on peut tracer un parallèle, qui ferait également du maitre une sorte d'archi-énonciateur, dans la mesure où il est amené à orchestrer et à hiérarchiser les points de vue issus du texte ou suscités par lui. Le parallélisme avec l'auteur se poursuit si l'on considère qu'il peut également y avoir plusieurs figures du maitre comme organisateur du savoir : cette «figure du maitre ", qui duplique celle de l'auteur, est en principe davantage ouverte, en fonction des activités et des réactions des apprenants. On peut penser cependant qu'une telle ouverture au dialogue présuppose que les enseignants aient bénéficié, lors de leur formation d'une approche épistémologique et historico-critique des savoirs, ce qui est loin d'être le cas. Autant dire que ces derniers ne sont pas toujours bien armés par l'institution comme par les éditeurs pour régler les questions d'hétérogénéité énonciative en lien avec la source et la nature des savoirs.

\section{La communication didactique entre la fin du mythe de l'unité du sujet parlant, la multiplicité des sources énonciatives et la synthèse de l'hétérogène}

On retrouve des préoccupations analogues en linguistique, autour de la fin du mythe de l'unicité et de l'autonomie du sujet parlant en linguistique. Ces remises en cause successives ont montré que les énoncés proférés par un sujet parlant n'avaient pas un énonciateur unique, qu'il s'agisse dusujet co-acteur, intersubjectif (Culioli) et/ou interactionnel (Bakhtine, Goffman); du sujet hétérogène, clivé par l'hétérogénéité constitutive ou hétérogénéité montrée (Authier-Revuz) ou par les rôles psychosociologiques interactionnels (Goffman); du sujet polyphonique, dont la parole est traversée par de multiples voix et points de vue (Ducrot) ${ }^{3}$. Ces diverses théorisations du dialogisme radical de la langue, de l'importance constitutive des relations intersubjectives et interactionnelles, ont pu jouer comme autant d'écrans qui rendaient opaque, sinon insane, la question de la responsabilité du locuteur et de celle des énonciateurs, sources de points de vue avec lesquels le locuteur/énonciateur est en adéquation, d'autant que, parallèlement, montait en puissance l'idée d'une coélaboration du sens, compte tenu de la nature profondément interactionnelle de la communication, qui est loin d'être propre à l'oral :

\footnotetext{
Il faut aller jusqu'à concevoir l'auditeur comme un vrai co-locuteur, l'interlocution comme la racine $\mathrm{du}$ sens, responsable du dialogisme réel du discours. L'interlocution dev[ient] plus radicale que l'intersubjectivité. [...] On parle avec l'autre et non pas seulement à l'autre. C'est moi qui parle mais c'est nous qui disons. (Jacques 1992 : 192)
}

La co-construction du sens et de la référence, fondamentale dans les interactions en face à face, joue aussi dans les textes, où la dynamique du sens passe par la mise en scène des énonciations rapportées ou la représentation de points de vue (Ducrot, 1984) qui ne sont pas des énonciations ${ }^{4}$, et elle traverse de part en part le processus interprétatif activé par les destinataires, à l'écrit comme à l'oral, sous des formes variables selon les situations et les genres de discours.

Toutefois, ce travail interprétatif ne peut opérer que sur la base d'une reconstruction, autrement de ce qui a été déconstruit: ce qui a été déconstruit, à juste titre, c'est le mythe de l'unicité du sujet parlant ${ }^{5}$ : ce qui devrait être reconstruit, c'est le principe d'une hiérarchisation des sources, qui se fonde sur la logique des échanges, et ce par- 
delà même la multiplicité des énonciateurs qui se font entendre sur la scène énonciative.

16 Se pose toujours la question d'une part de l'inscription du scripteur dans son propre texte, de son positionnement par rapport à ses sources, aux autorités, de sa place et de son point de vue par rapport à des thèses antagonistes, des principes à la source des classements qu'il opère (ou non), d'autre part de la place qu'il réserve à son destinataire dans son propre discours. Bornons-nous au premier point : l'inscription du locuteur (ou du scripteur) dans son discours soulève de nombreuses difficultés :

- articulation des concepts de locuteur et d'énonciateur, compris tantôt comme un doublon de locuteur, tantôt comme une instance intradiscursive disjointe du locuteur (cf. articles consacrés à ces notions dans Charaudeau et Maingueneau 2002, Rabatel 2004) ; - effacement des sources énonciatives et naturalisation des points de vue. La question concerne d'abord les sources énonciatives qui renvoient à des êtres discursifs incarnés, à des sujets parlants dotés d'une visibilité sur la scène sociale et/ou culturelle ou scientifique. Mais elle concerne aussi un certain effacement de ces sources (sur le plan linguistique, mais pas sur le plan épistémologique), lorsque la pratique didactique, aidée en cela par la dimension cumulative du savoir et les contraintes parfois contradictoires de l'Institution, s'astreint non seulement à une grande neutralité (apparente) dans la présentation de points de vue antagonistes sur un même sujet, mais encore va jusqu'à naturaliser les savoirs qui ont pu faire l'objet de luttes scientifiques acharnées dans le champ scientifique. Le gommage des enjeux n'est guère de nature à faire comprendre les objectifs et les limites de telle ou telle théorie, il donne au contraire une image réductrice de notions complexes, au détriment de la réalité et des analyses de construction de la réalité, et ce dans toutes les disciplines d'enseignement, lorsqu'il s'agit de référer à des courants politiques (le libéralisme, le catholicisme social, la social-démocratie, l'anarchie, le nationalisme, le pacifisme), à des esthétiques (le romantisme, le surréalisme, le dodécaphonisme, le fauvisme), à des systèmes ou catégories philosophiques (l'idéalisme, le scepticisme, l'existentialisme) ou à des théories scientifiques (l'évolutionnisme, le structuralisme, la pragmatique, la physique quantique) ${ }^{6}$. Cependant, il faut reconnaître que l'historicisation des enjeux et des points de vue, si elle est gage d'une prise en compte de la complexité dans les manuels, pose des problèmes redoutables: d'une part, en effet, elle s'oppose partiellement à la cohérence du discours primaire, ou au moins risque de la rendre moins lisible ; d'autre part, elle va à l'encontre du statut didactique du manuel qui, par définition, doit fournir des repères. La solution, s'il y en a une, réside selon nous dans une meilleure explicitation - pour le maitre comme pour l'élève - des choix théoriques effectués, et le renvoi à des discours secondaires grâce à l'appareil paratextuel ;

19 - questions de prise en charge énonciative. La prise en charge (ou responsabilité) est un phénomène par défaut qui résulte des maximes conversationnelles, notamment du principe de sincérité. Ce qu'on asserte, on l'assume, en tant que locuteur/énonciateur primaire. C'est pourquoi la prise en charge ne requiert pas de marques spécifiques, à la différence de la non-prise en charge (conditionnel, modalisateurs épistémiques, dimension axiologique du lexique, discours rapportés, cadratifs, etc.). Or, dans les manuels qui juxtaposent des sources différentes, la prise en charge se pose crucialement, à la fois pour le discours primaire et pour les discours enchâssés, émanant d'autres instances énonciatrices. Car si l'énonciateur primaire n'est pas responsable du contenu des discours enchâssés, du moins est-il responsable de leur 
sélection et de leur éclairage : c'est notamment à cette aune que les discours primaires qui accompagnent les sources doivent être évalués.

Ces questions fondamentales sont traitées sous des angles très différents par les contributions réunies dans ce numéro, qui vise à dessiner la figure complexe de l'auteur en didactique. Un premier groupe d'articles s'intéresse prioritairement aux problèmes posés par la didactisation de notions linguistiques. Françoise Boch et Francis Grossmann, à travers une enquête portant sur les manuels de 3ème, analysent la manière dont sont présentées les notions issues des théories de l'énonciation, aujourd'hui intégrées aux programmes officiels du collège. Le bilan est contrasté : alors que la linguistique de l'énonciation conduit parfois à redonner du sens à des notions traditionnelles (Voir le discours rapporté), dans d'autre cas, l'appareil terminologique introduit a tendance à obscurcir les phénomènes décrits plutôt qu'à les éclairer. Etre un auteur, en didactique, exige donc d'opérer des choix clairs, parfois drastiques dans un champ théorique, en fonction de besoins clairement identifiés. Alain Rabatel, dans une étude de cas portant sur un manuel de $\mathrm{CE} 2^{7}$, montre que la présence d'un discours intégrateur fort n'est pas en elle-même suffisante pour proposer une approche crédible au plan didactique. Si l'effacement des sources savantes se justifie dans un manuel, il n'en est pas moins nécessaire, selon lui, d'expliciter, pour les élèves mêmes, les " grands cadres d'analyse sémantiques, morphologiques, syntaxiques et énonciatifs à partir desquels un point de langue est appréhendé ». C'est précisément la capacité de passer d'un point de vue à l'autre qui permet aux élèves de comprendre l'intérêt de certaines procédures formelles, tout en leur évitant l'utilisation aveugle des tests. Frédérique Sitri pose, quant à elle, la question de l'intégration d'une notion " nouvelle », celle de registre (utilisée au lycée pour caractériser les textes littéraires, et non, comme au collège, les niveaux de langue). Cette notion semble très proche de celle de tonalité, que continuent à mobiliser les manuels, mais ce n'est pas tant la cohérence terminologique qui est en cause ici que l'impensé théorique qu'elle révèle, dans le défaut d'articulation des registres avec les notions connexes de tonalité, genre, type de textes.

21 Un deuxième groupe de contributions prend en compte plus nettement la question des supports utilisés, ainsi que celle de la situation de communication didactique. Isabelle Laborde-Milaa s'intéresse, à partir d'un corpus de manuels, à la «fiche", unité textuelle qui y apparaît fréquemment aujourd'hui. L'«imaginaire techniciste » sur lequel elle se fonde, associé aux fonctions pragmatiques qu'elle semble convoquer, ne doit pas masquer les difficultés que pose son intégration. La plasticité du genre semble en effet autoriser des pratiques textuelles extrêmement hétérogènes, qui conduisent parfois, paradoxalement, à opacifier ou à brouiller la figure de l'auteur plutôt qu'à la préciser. Anthippi Potolia analyse de son côté un corpus de cédéroms de vulgarisation scientifique, en montrant les évolutions de l'écriture multimédia : après une période durant laquelle le foisonnement des images et la liberté de la navigation de l'utilisateur ont été privilégiées, l'accent est mis aujourd'hui sur les procédures de structuration du savoir et une certaine sobriété visuelle. Robert Bouchard et Chantal Parpette s'intéressent, quant à eu, aux reformulations opérées par l'enseignant lui-même, lors de cours magistraux dans l'enseignement supérieur. On peut considérer en effet qu'il s'agit bien là également d'une forme de transposition didactique, même si, comme le remarquent les auteurs, la transposition est plus « courte ", les sources savantes étant cette fois fournies. Cependant, l'interaction face à face, quand bien même elle reste à 
sens unique, autorise des excursus qui favorisent l'émergence d'un auteur plus engagé, précisant les enjeux du savoir transmis dans l'espace public.

Il nous a semblé intéressant, étant donné l'actualité du thème de la mémoire et de la formation du citoyen, de regrouper, pour finir, deux contributions qui analysent la figure de l'auteur dans le cours d'histoire. Anne Leclaire-Halté analyse le positionnement énonciatif de l'enseignant - archi-énonciateur qui doit "orchestrer » les points de vue émanant de sources hétérogènes - lors d'une séquence d'histoire au cycle 3 , à travers la comparaison de deux documents iconographiques représentant le baptême de Clovis. Elle montre comment la mise au premier plan d'objectifs méthodologiques (attirer l'attention des élèves sur le fait que l'on ne peut faire une confiance aveugle aux documents) aboutit de fait à minorer la réflexion proprement historique, tout en traitant de manière insatisfaisante le point de méthode choisi.

Enfin Françoise Lantheaume, étudie d'un point de vue sociologiquex- qui n'ignore pas les problématiques linguistiques enæœuvre dans ce numéro - la manière dont a été traitée la question de la colonisation. Son analyse, basée sur un large corpus (73 manuels de lycée publiés entre 1937 et 2004), débouche sur le constat d'une injonction paradoxale : les manuels doivent tout à la fois assurer leur mission de transmission patrimoniale et le développement de l'esprit critique. Cette tension entre des objectifs contradictoires renvoie, selon nous, à une difficulté plus générale, et urgente : redéfinir la place d'auteur dans le champ didactique.

\section{BIBLIOGRAPHIE}

\section{Références bibliographiques}

Barthes, R. (1970)æ: L'ancienne rhétorique, Communications, 16, 172-229.

Boch, F. \& Grossmann, F. (2002)æ: Se référer au discours d'autrui, quelques éléments de comparaison entre experts et néophytes, Enjeux, 54, 41-51.

Charaudeau, P. \& Maingueneau, D. (2002)æ: Dictionnaire d'analyse du discours, Le Seuil, Paris.

De Certeau, M. (1990)๙: L'invention du quotidien, Gallimard, Paris.

Ducrot, O. (1984)æ: Le dire et le dit. Minuit, Paris.

Collinot, A. \& Petiot, G. (1998)æ: Avant-propos, Les Carnets du Cediscor, 5, 9-12.

Collinot, A. \& Petiot, G. (1998)®: Table ronde, Les Carnets du Cediscor, 5, 137-140.

Delesalle, S. (1998) $₫$ : Autour de la notion de discours : théories, manuels, programmes, Les Carnets du Cediscor, 5, in A. Collinot \& G. Petiot (éd.), 31-38.

Elalouf, M.-L. (1998)æ: L'énonciation dans les nouveaux sujets du baccalauréat, Les Carnets du Cediscor, 5, 115-133.

Foucault, M. (2001a)æ: Dits et écrits 1, Paris, Gallimard.

Foucault, M. (2001b)æ: Dits et écrits 2, Paris, Gallimard. 
Grossmann, F. (2002) : Les modes de référence à autrui chez les experts : l'exemple de la revue Langages, Faits de Langues,19, 255-261.

Grossmann, F. (2003)æ: Du discours rapporté au discours autorisé, le maniement des noms d'auteur dans l'article en Sciences humaines, Estudios de Lengua y literatura francesas, 14, 9-31, Université de Cadix.

Maingueneau, D. (1990)æ: Pragmatique pour le texte littéraire, Paris, Bordas.

Philippe, G. (2000) $₫$ : Centre énonciatif et centre interprétatif. L'analyse linguistique et l'interprétation du roman, Études romanesques,6, 37-52.

Philippe, G. (2002) $₫$ : L'appareil formel de l'effacement énonciatif et la pragmatique des textes sans locuteur, in R. Amossy (dir.)æ: Pragmatique et analyse des textes, Université de Tel-Aviv, 17-34. Rabatel, A. (2004)æ: L'effacement énonciatif dans les discours rapportés et ses effets pragmatiques, Langages, 156, 3-17.

Rabatel, A. (2005) : La part de l'énonciateur dans la construction interactionnelle des points de vue, Marges linguistiques,9, 115-136.

Vion, R. (1998) $₫$ : Du sujet en linguistique, in R. Vion (dir.) $₫$ : Les sujets et leurs discours. Énonciation et interaction. Publications de l'université de Provence, 189-202.

\section{AUTEURS}

\section{ALAIN RABATEL}

Université Lumière-Lyon 2, Icar, Umr 5191

\section{FRANCIS GROSSMANN}

Université Stendhal, Grenoble3, Lidilem, E. A. 609 